\title{
Image dehazing based on dark channel prior and brightness enhancement for agricultural monitoring
}

\author{
Xiuyuan Wang ${ }^{1,2,3}$, Chenghai Yang ${ }^{4}$, Jian Zhang ${ }^{5}$, Huaibo Song ${ }^{1,2,3 *}$ \\ (1. College of Mechanical and Electronic Engineering, Northwest A\&F University, Yangling 712100, Shaanxi, China; \\ 2. Key Laboratory of Agricultural Internet of Things, Ministry of Agriculture, Yangling 712100, Shaanxi, China; \\ 3. Shaanxi Key Laboratory of Agricultural Information Perception and Intelligent Service, Yangling, Shaanxi 712100, China; \\ 4. USDA-ARS Southern Plains Agricultural Research Center, 3103 F and B Road, College Station, Texas 77845, USA; \\ 5. College of Resource and Environment, Huazhong Agricultural University, Wuhan 430070, China)
}

\begin{abstract}
Obtaining clear and true images is a basic requirement for agricultural monitoring. However, under the influence of fog, haze and other adverse weather conditions, captured images are usually blurred and distorted, resulting in the difficulty of target extraction. Traditional image dehazing methods based on image enhancement technology can cause the loss of image information and image distortion. In order to address the above-mentioned problems caused by traditional image dehazing methods, an improved image dehazing method based on dark channel prior (DCP) was proposed. By enhancing the brightness of the hazed image and processing the sky area, the dim and un-natural problems caused by traditional image dehazing algorithms were resolved. Ten different test groups were selected from different weather conditions to verify the effectiveness of the proposed algorithm, and the algorithm was compared with the commonly-used histogram equalization algorithm and the DCP method. Three image evaluation indicators including mean square error (MSE), peak signal to noise ratio (PSNR), and entropy were used to evaluate the dehazing performance. Results showed that the PSNR and entropy with the proposed method increased by $21.81 \%$ and $5.71 \%$, and MSE decreased by $40.07 \%$ compared with the original DCP method. It performed much better than the histogram equalization dehazing method with an increase of PSNR by $38.95 \%$ and entropy by $2.04 \%$ and a decrease of MSE by $84.78 \%$. The results from this study can provide a reference for agricultural field monitoring.
\end{abstract}

Keywords: agricultural monitoring, image dehazing, monitoring image, dark channel prior (DCP), brightness promoting DOI: $10.25165 /$ j.ijabe.20181102.3357

Citation: Wang X Y, Yang C H, Zhang J, Song H B. Image dehazing based on dark channel prior and brightness enhancement for agricultural monitoring. Int J Agric \& Biol Eng, 2018; 11(2): 170-176.

\section{Introduction}

Accurate agricultural monitoring systems are of great importance for conserving resources, reducing pollution, improving the environment and efficiency ${ }^{[1]}$. With the rapid development of machine vision technology, high-definition images have been increasingly used for accurate monitoring of crop growth status and field conditions. However, under the influence of fog, haze and other adverse weather conditions, images are usually degraded. The degraded images may become grayish white with low contrast $^{[2]}$. They also contain less information than the images captured in ideal conditions, making difficulties for feature extraction and other usage. It is important to dehaze images taken under the conditions of fog and haze for environmental improvement, disaster prevention and mitigation, and yield

Received date: $2017-03-21 \quad$ Accepted date: $2017-08-28$

Biographies: Xiuyuan Wang, Bachelor, research interests: image processing, Email: 15929321225@163.com; Chenghai Yang, PhD, Professor, research interests: development and evaluation of remote sensing technologies for detecting and mapping crop pests for precision chemical applications, Email: chenghai.yang@ars.usda.gov; Jian Zhang, PhD, Associate Professor, research interests: agricultural remote sensing, field high-throughput plant phenotyping and analysis based on UAV platform, Email: jz@mail.hzau.edu.cn.

*Corresponding author: Huaibo Song, PhD, Associate Professor, research interests: image processing. College of Mechanical and Electronic Engineering, Northwest A\&F University, 22 Xinong Road, Yangling 712100, Shaanxi, China. Tel: +86-29-87092391, Email: songhuaibo@nwsuaf.edu.cn. monitoring ${ }^{[3]}$. Therefore, image dehazing has a wide range of practical applications and has received increasing attention ${ }^{[4,5]}$.

At present, many researchers in various fields have carried out studies on image dehazing. Related research methods can be divided into two categories: image enhancement and image restoration $^{[6]}$. Image enhancement improves the image quality by enhancing the degraded image, and histogram equalization was commonly used in this category. For example, Zhang et al. ${ }^{[7]}$ achieved image dehazing based on the histogram equalization; Yang et al. ${ }^{[8]}$ proposed an image dehazing algorithm with luminance mapping; Li et al. ${ }^{[9]}$ proposed an infrared image enhancement method based on the haze removal model, combining the characteristic of the infrared image to improve the visible light dehazing enhancement method. These methods can enhance the contrast of the images, but for more complex images, they may cause the loss of image information, resulting in image distortion ${ }^{[2]}$. These methods did not meet the requirements for areas where image accuracy and clarity are demanded. Based on the method of image restoration, the image degradation model was established according to the law of atmospheric scattering, and by reversing the deduction, the original image was restored ${ }^{[10,11]}$. For example, He et al. ${ }^{[12]}$ proposed a novel haze removal algorithm based on dark channel prior (DCP). This algorithm could add the depth of field information to make the image more realistic. Many improvements have been made to the method ${ }^{[13-19]}$. Hu et al. ${ }^{[20]}$ proposed an image dehazing algorithm based on an atmosphere scattering approximation model; Ding et al. ${ }^{[21]}$ proposed an 
effective DCP algorithm based on image dehazing using quad trees; Wang et al. ${ }^{[22]}$ proposed an improved single image dehazing algorithm using DCP; Wang et al. ${ }^{[23]}$ used a physical model to explain the objective rationality of the DCP method.

Although these improved methods based on DCP algorithm have achieved good results in diverse environmental conditions, limited research has been conducted for agricultural field monitoring. There are a couple of reasons for this. First, agricultural field monitoring is in a complex non-structural environment, which is greatly affected by geographical and climate factors. Especially in the foggy and hazy weather, image enhancement technology cannot effectively remove the fog, resulting in the loss of image information and image distortion. Therefore, this technology cannot be applied in agricultural monitoring. Second, traditional image dehazing methods cannot add image depth information, which results in the lack of necessary three-dimension sense. To address the image dehazing needs for agricultural monitoring systems, it is necessary to identify appropriate image dehazing software and techniques. The objective of this study was to propose a new image dehazing method that can meet the imaging needs of agricultural monitoring systems.

\section{Materials and methods}

\subsection{Materials}

\subsubsection{Imaging system}

All the images were captured using a webcam. The frame of the image acquisition system can be seen in Figure 1. The system was developed using Microsoft Visual Studio 2008 (Microsoft Corporation, Redmond, Washington, USA). The YW7600 high-definition net cam (Yiwei Ruichuang Science and Technology Limited Company, Shenzhen, Guangdong, China) with a resolution of $704 \times 576$ pixels was selected as the webcam. The rotation range of the camera is $0-360^{\circ}$ horizontal and $0-90^{\circ}$ vertical, and the frame rate is $25 \mathrm{fps}$. Its main functions include remote connection, line-of-sight stacking, and pan/tilt/zoom (PTZ) control. Based on this webcam platform, real-time monitoring of all farmland within the range covered by the camcorder can be realized.

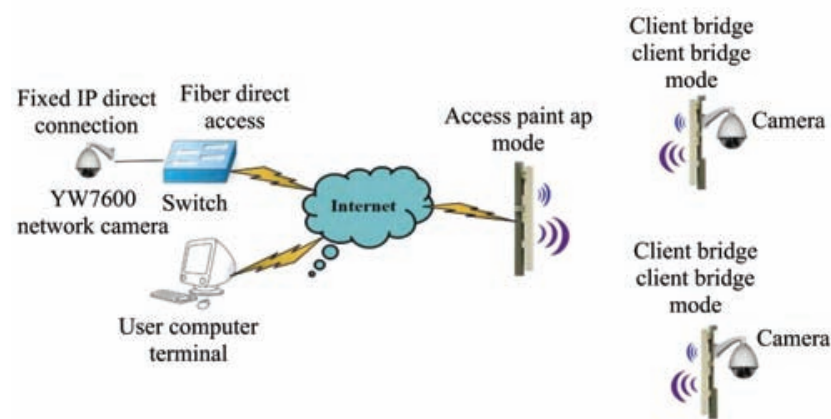

Figure 1 Agricultural network access platform

All of the algorithms in this study were carried out on a laptop with the Intel i5 processor, 4G memory equipped with Windows 7 system. The algorithm running environment was Matlab 2015a (MathWorks, Natick, Massachusetts, USA).

\subsubsection{Study sites}

In order to verify the effect of image dehazing in this study, 20 images groups captured in different days were selected from the Baishui Apple Test Station, the Shanyang Chestnut Walnut Test and Planting Station, and the Gaoping Modern Agriculture Park, all located in Shaanxi Province. The sunny haze-free images from three test stations in different days at different times were selected as reference images to verify the effect of the image dehazing algorithm. The basic information of the test images can be seen in Table 1.

Table 1 Basic information of the test images

\begin{tabular}{|c|c|c|c|c|c|}
\hline No. & Test stations & $\begin{array}{l}\text { Number of } \\
\text { images }\end{array}$ & $\begin{array}{l}\text { Haze } \\
\text { level }\end{array}$ & & Time \\
\hline \multirow{5}{*}{1} & \multirow{5}{*}{$\begin{array}{c}\text { Baishui Apple Test } \\
\text { Station }\end{array}$} & \multirow{5}{*}{10} & \multirow{5}{*}{ Serious } & Group1: & $\begin{array}{l}2016-12-2911: 18: 25 \\
2017-01-01 \quad 08: 43: 44\end{array}$ \\
\hline & & & & Group2: & $\begin{array}{l}2016-12-16 \text { 11:52:33 } \\
2016-12-15 \text { 15:35:04 }\end{array}$ \\
\hline & & & & Group3: & $\begin{array}{l}2016-12-1607: 49: 14 \\
2016-12-16 \quad 11: 52: 33\end{array}$ \\
\hline & & & & Group4: & $\begin{array}{l}2016-12-15 \text { 09:56:08 } \\
2016-12-15 \text { 13:24:36 }\end{array}$ \\
\hline & & & & Group5: & $\begin{array}{l}2017-02-2312: 00: 51 \\
2017-02-2310: 07: 15\end{array}$ \\
\hline \multirow[t]{2}{*}{2} & $\begin{array}{c}\text { Shanyang Chestnut } \\
\text { Walnut Test and }\end{array}$ & \multirow[t]{2}{*}{4} & \multirow{2}{*}{ General } & Group6: & $\begin{array}{l}2016-12-15 \text { 13:23:25 } \\
2016-12-15 \text { 09:55:36 }\end{array}$ \\
\hline & Planting Station & & & Group7: & 2016-12-31 07:13:57 \\
\hline \multirow{3}{*}{3} & \multirow{3}{*}{$\begin{array}{l}\text { Gaoping Modern } \\
\text { Agriculture Park }\end{array}$} & \multirow{3}{*}{6} & \multirow{3}{*}{ General } & Group8: & $\begin{array}{l}2016-12-3107: 38: 38 \\
2017-02-04 \quad 15: 04: 12\end{array}$ \\
\hline & & & & Group9: & $\begin{array}{l}2017-01-01 \quad 16: 37: 58 \\
2017-02-04 \quad 15: 04: 12\end{array}$ \\
\hline & & & & Group10: & $\begin{array}{l}2017-02-23 \quad 10: 05: 43 \\
2017-01-2010: 23: 14\end{array}$ \\
\hline
\end{tabular}

Portions of the test images obtained are shown in Figure 2. Figure 2a shows an example image when the test station was sunny without haze and Figure 2b shows an example image with fog and haze in the test station. The average light intensity of the three test stations was low, whereas the haze in the Baishui Apple Test Station was more serious. Since several groups of images were affected by haze, they were all blurred, making it difficult to observe. Moreover, because of the lack of light in winter, foggy haze in some areas was serious. Even without haze or with slight haze, image clarity was reduced by the impact of light.

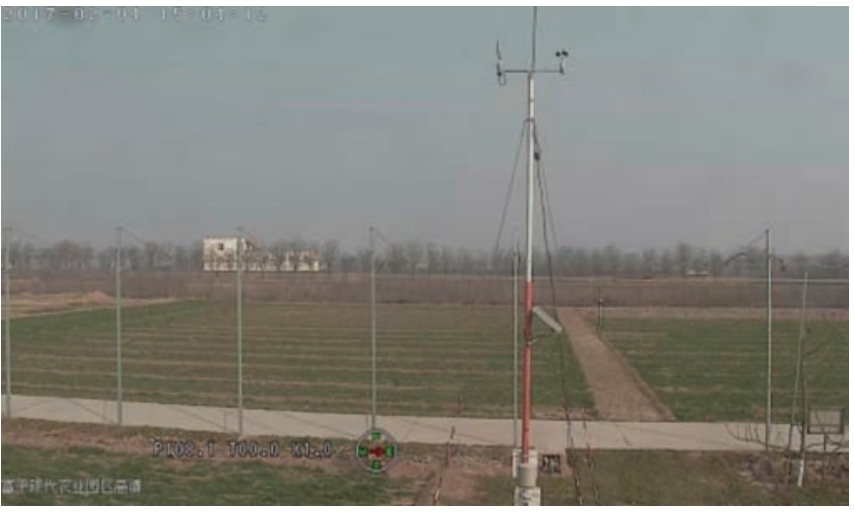

a. Image without haze

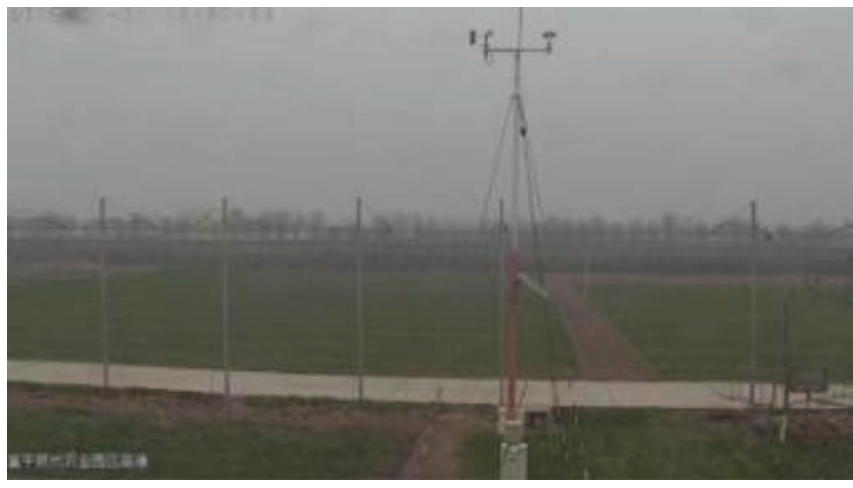

b. Degraded image with haze

Figure 2 Comparison of images in the Gaoping Modern Agricultural Park in Shaanxi 


\subsection{Methods}

\subsubsection{Image dehazing algorithm based on DCP}

According to Koschmieder's theory ${ }^{[24]}$, in computer vision and computer graphics, the fog pattern formation model can be described by Equation (1):

$$
I(x)=J(x) t(x)+A(1-t(x))
$$

where, $x$ refers to a pixel block of the image; $I$ refers to intensity of the observed image; $J$ refers to intensity of the scene light; and $A$ refers to global atmospheric light composition. $t$ is used to describe the non-scattered part of the light through the media transmission to the camera.

The goal of image dehazing is to derive $J$ from $A, t$ and $I$. Since it cannot be obtained directly by Equation (1), He et al. ${ }^{[12]}$ proposed an image dehazing method based on DCP. Equation (1) can be changed into Equation (2):

$$
I^{c}(x) / A^{c}=t(x) J^{c}(x) / A^{c}+1-t(x)
$$

where, $A^{c}$ represents each channel of the global atmosphere light component; $I^{c}$ represents the intensity of the image observed for each channel; and $J^{c}$ represents light intensity of each channel.

For any input image $\boldsymbol{J}$, its dark channel $\boldsymbol{J}^{\text {dark }}$ is expressed by Equation (3):

$$
\boldsymbol{J}^{\text {dark }}(x)=\min _{y \in \Omega(x)}\left(\min _{c \in\{r, g, b\}} \boldsymbol{J}^{c}(y)\right)
$$

where, $\boldsymbol{J}^{c}$ represents each channel of the color image and $\Omega(x)$ represents a window centered on pixel $X$.

The DCP theory points out that $\boldsymbol{J}$ 's dark primary colors $\boldsymbol{J}^{\text {dark }}$ should tend to 0. According to the above definition, Equation (4) is obtained:

$$
\boldsymbol{J}^{\text {dark }}(x)=\min _{c}\left[\min _{y \in \Omega(x)}\left(\boldsymbol{J}^{c}(y)\right)\right]=0
$$

Therefore, Equation (5) can be obtained:

$$
\min _{y \in \Omega(x)}\left[\min _{c}\left(\boldsymbol{J}^{c}(y) / A^{c}\right)\right]=0
$$

Assuming that the transmittance $t(x)$ is constant in each window and defined as $\ell(0 x)$. And the value $A$ is given. Then the minimum value is calculated twice for Equation (2) to obtain Equation (6) and Equation (7):

$$
\begin{gathered}
\min _{c}\left(I^{c}(x) / A^{c}\right)=\mathfrak{f}(0 x) \min _{c}\left(J^{c}(x) / A^{c}\right)+1-\mathfrak{l}(0 x) \\
\min _{y \in \Omega(x)}\left(\min _{c} I^{c}(y) / A^{c}\right)=\mathfrak{f}(0 x) \min _{y \in \Omega(x)}\left(\min _{c} J^{c}(y) / A^{c}\right)+1-\mathfrak{l}(0 x) \\
\text { Substituting Equation (5) into the Equation (7), Equation (8) }
\end{gathered}
$$
can be obtained:

$$
\mathcal{f}(0 x)=1-\omega \min _{y \in \Omega(x)}\left(\min _{c} I^{c}(y) / A^{c}\right)
$$

where, $f(0 x)$ is the estimated value of transmittance; $\omega$ is the information with fog, whose assignment affects the final image of the dehazing effect. It can make the image look more real, with the depth of field effect and it generally takes the value of 0.95 .

If the value of the pixel transmittance $t(x)$ of a pixel in the transmission map is small, it will cause the value of $J$ to be too large, so that the whole image is excessively white. Therefore, a threshold value $t_{0}$ can be set. When $t(x)$ is less than $t_{0}$, let $t=t_{0}$. According to Equation (1), if the threshold is set to be $t_{0}$, the final recovery Equation (9) will be:

$$
J(x)=\frac{I(x)-A}{\max \left(t(x), t_{0}\right)}+A
$$

where, $t_{0}$ generally takes the value 0.1 .

\subsubsection{Improved method}

The image dehazing method based on DCP would result in images that are darker than the actual images, and the effect of the sky part would not be good enough. To solve the problem, this study improved the above algorithm. The dehazed image $J(x)$ was highlighted and the sky section was processed. The formula for the image highlighted is shown in Equation (10):

$$
T(x)=J(x)+(1-J(x)) \times J(x)
$$

where, both $J$ and $T$ are normalized images. Their values belong to $[0,1] . \quad T$ is the highlighted image and $J(x)$ is the dehazed image.

In order to control the bright effect of the dehazed image, a control parameter $K$ is introduced. It can control the strength of brightness increase as shown in Equation (11). The larger the value of $K$ is, the brighter of the image will be. When $K=0$, the image is not highlighted. When $K=1$, the image is fully highlighted.

$$
T(x)=J(x)+(1-J(x)) \times J(x) \times K
$$

To handle the sky element, a controllable parameter $M$ was added to adjust the transmittance at each pixel:

$$
F(x)=\frac{T(x)-A}{\min \left(\max \left(\frac{M}{|T(x)-A|}, 1\right) \cdot \max \left(t(x), t_{0}\right), 1\right)}+A
$$

where, the value of $M$ is showed in Equation (13):

$$
\left\{\begin{array}{l}
M \neq 0\left\{\begin{array}{l}
|T(x)-A| \leq M \\
|T(x)-A|>M
\end{array}\right. \\
M=0
\end{array}\right.
$$

Generally, the value of atmospheric light $A$ and the sky part is very close. The area that follows DCP is far from the sky. So the threshold $M$ is introduced to detect whether the region is sky. If $M$ is not 0 , when $|T(x)-A| \leq M$, it means that $\mathrm{I}(x)$ is close to the atmospheric light $A$. So this area may be the sky. Then the transmittance increased. If $|T(x)-A|>M$, it is considered to be consistent with DCP, so the transmittance is unchanged. If $M$ is 0 , it corresponds to the original transmittance formula. Experiments have showed that the general value of $M$ is 0.1 .

Based on the theory above, the dehazed image based on DCP is first highlighted according to Equation (11), then the sky area is processed according to Equation (12). Several images from the Shanyang Chestnut Walnut Test Station captured on December 31, 2016 were selected as an example. The dehazing effect is shown in Figure 3. Figure 3a shows the original image with fog. It can be seen that the information in the image was greatly degraded by the influence of haze. Figure $3 \mathrm{~b}$ shows the dehazed image by directly applying DCP. The image was generally dim by using this method. Figure $3 \mathrm{c}$ shows the highlighted image by adding image enhancement technology. The image's brightness was significantly enhanced, but the sky area was seriously distorted. Figure $3 \mathrm{~d}$ shows the result after processing the sky element. The final processed image was closer to the real sky. So the overall effect was better.

\subsection{Evaluation indicators of dehazing effect}

In order to examine the performance of the proposed method objectively, three image indicators including mean square error (MSE), peak signal to noise ratio (PSNR), and entropy were used to evaluate the image processing effect ${ }^{[6]}$. To verify the performance of the proposed method, it was compared with He's DCP image dehazing method and the conventional histogram equalization method.

MSE is the mean square error, indicating the difference between the processed image and the reference image. The lower the value is, the closer the image is to the reference image, as shown in Equation (14): 


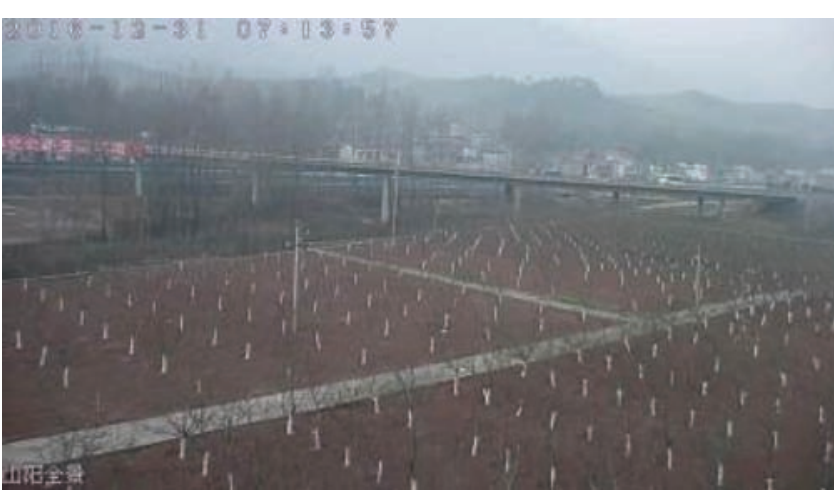

a. Original image with fog

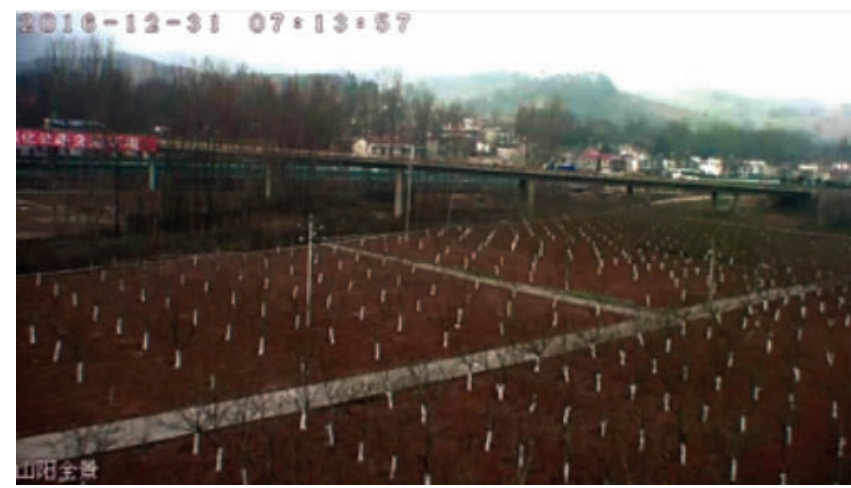

b. Direct image dehazing with DCP

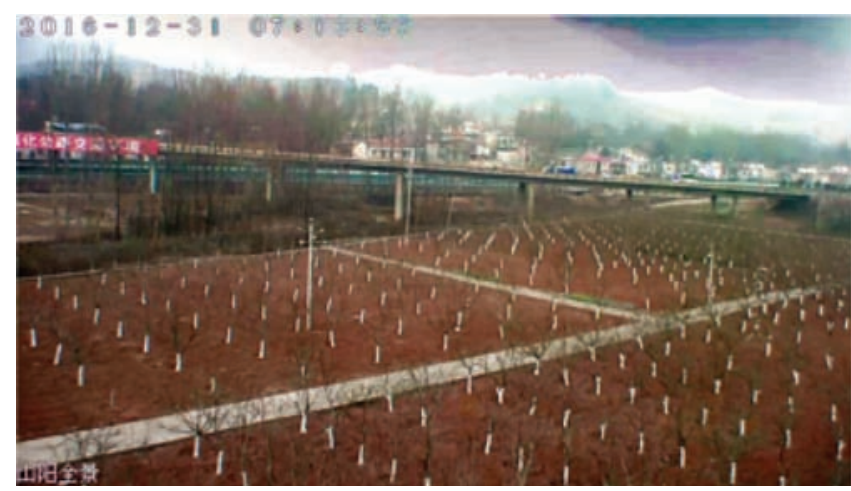

c. Highlighted image with enhancement

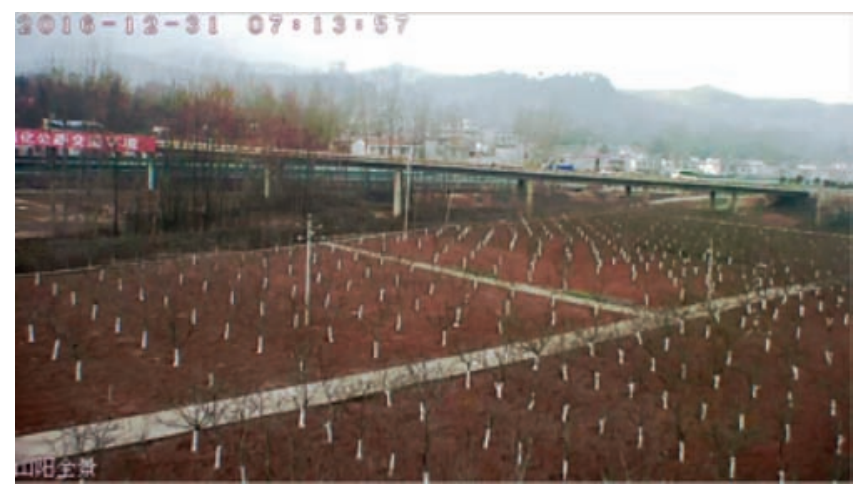

d. Image with processed sky element

Figure 3 Comparison of dehazed images with DCP and the proposed method

$$
\mathrm{MSE}=\sum_{x=1}^{N_{x}} \sum_{y=1}^{N_{y}}(f(x, y)-\hat{f}(x, y))^{2} / N_{x} N_{y}
$$

where, $f(x, y)$ is the pixel value of each pixel block for the image. In this paper, it represents the pixel value of the corresponding reference image; $\hat{f}(x, y)$ is the value of each pixel block for the processed image. $N_{x}$ and $N_{y}$ represent the number of rows and columns of the image matrix respectively.

PSNR is the peak signal to noise ratio, which indicates the ratio of the maximum value to the noise of the image signal. The larger the value is, the smaller the interference of the image by the noise and the better the image quality is. The formula is shown in Equation (15):

$$
\text { PSNR }=20 \cdot \log _{10}(255 / \text { MSE })
$$

Entropy is the two-dimensional information entropy of the image. The formula is shown in Equation (16):

$$
\mathrm{H}=\sum_{i=0}^{255} p_{i j} \log p_{i j}
$$

where, $i$ represents grayscale value and $j$ represents the mean of horizontal gray scale. The range for $i$ and $j$ is [0,255]. $p_{i j}$ represents the probability that the pixel block of gray scale $i$ is adjacent to the pixel block with gray scale $j$. The formula is shown in Equation (17):

$$
p_{i j}=f(i, j) / N^{2}
$$

where, $f(i, j)$ is the frequency of the characteristic binary $(i, j)$. The two-dimensional information entropy of the image reflects the average amount of information in the image, the value range is $[0,8]$. The larger the value is, the more uniform the gray value of the image is.

\section{Results and analysis}

\subsection{Results}

In order to verify the effectiveness of the proposed method, the test images were processed and compared the obtained results with image dehazing based on DCP and histogram equalization. The comparison results are shown in Figure 4. The images in column (a) are the original images with fog. The images in columns (b), (c) and (d) are the dehazed images based on DCP, histogram equalization, and the proposed method respectively. The images in column (e) are the reference images. For the processed images using the proposed method, $t_{0}$ in Equation (7) was set to 0.1 and $\omega$ in Equation 6 was set to 0.95 . The three indicators for the three dehazing methods are shown in Figure 5.

\subsection{Analysis}

\subsubsection{Comparison of evaluation indicators}

As is illustrated in Figure 5, values of 3 evaluation indicators from 9 groups of images are calculated using 3 different methods. Then the evaluation indicators of the 9 images obtained by each method are averaged. According to the data, it can be seen that the MSE for the proposed method was $40.07 \%$ lower than that for the DCP method and $84.78 \%$ lower than that for the histogram equalization method. The PSNR for the proposed method was $21.81 \%$ higher than that for DCP and $38.95 \%$ higher than that for histogram equalization. The proposed method had entropy that was $5.71 \%$ higher than DCP and $2.04 \%$ higher than histogram equalization. All three indicators show the superiority of the proposed method.

3.2.2 Comparison between proposed method and two other methods

In Figure $4 b$, the reconstructed images obtained by the direct dehazing DCP method were much darker than the original images. The dehazing effect was different from the human visual effect, and there was an obvious deficiency in the treatment of sky area. It can also be seen from Figure 5a that the average MSE obtained by the direct DCP method was larger than that of the proposed method in this paper. As shown in Figure 4d, the brightness of 
the images was obviously enhanced after the images were brightened and the sky area was processed. The non-sky area was significantly improved. The final recovered images had a high contrast, uniform brightness, and natural sky in line with human visual effects. The proposed method can be used for image dehazing for agricultural monitoring.

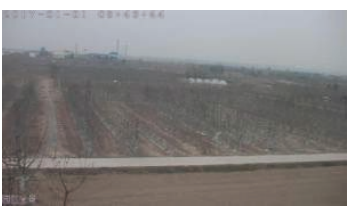

a1

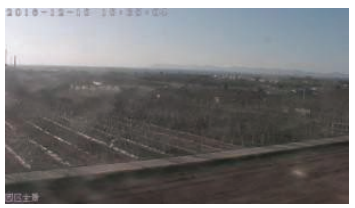

a2

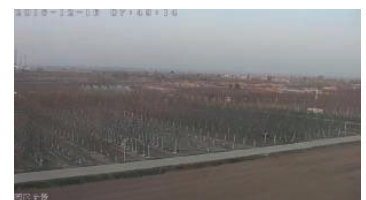

a3

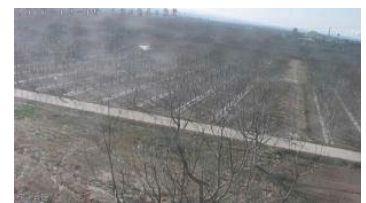

a4

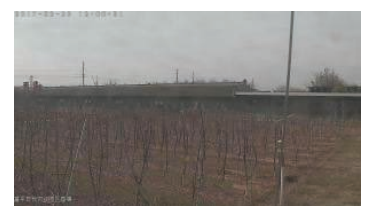

a5

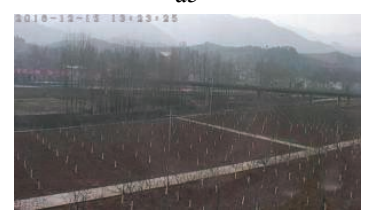

a6

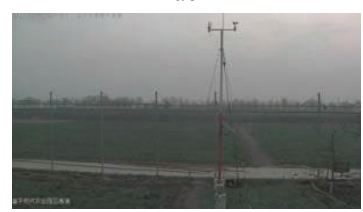

a7

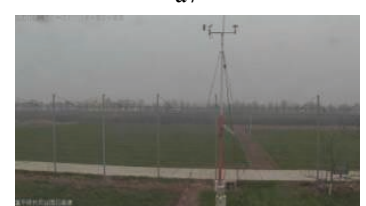

a8

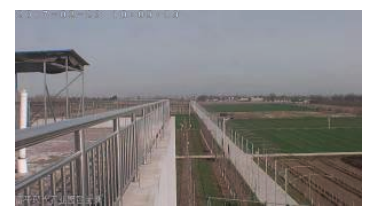

a9

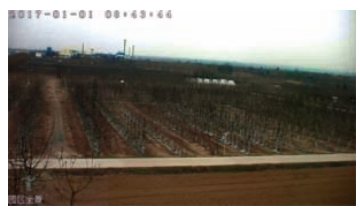

b1

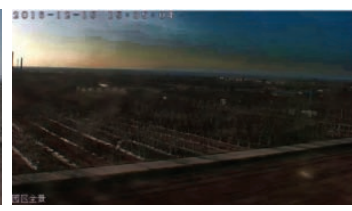

b2

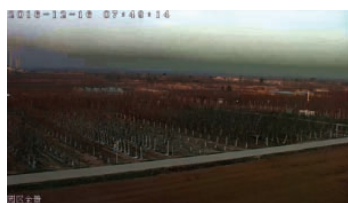

b3

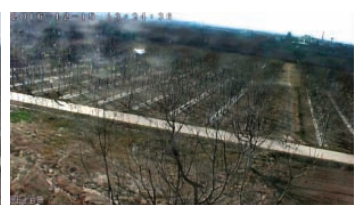

b4

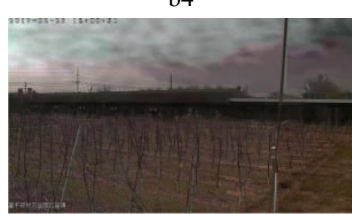

b5

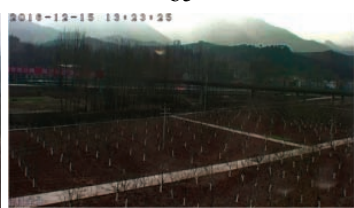

b6

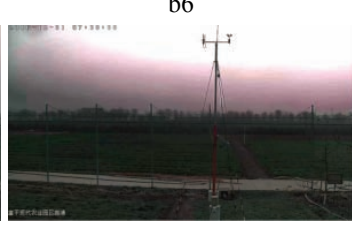

b7

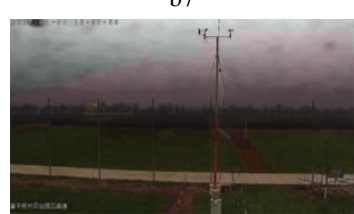

b8

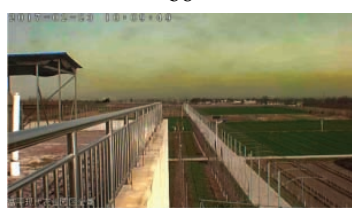

b9

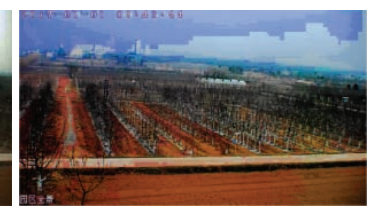

c1

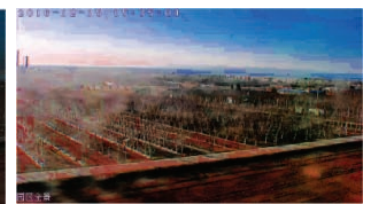

c2

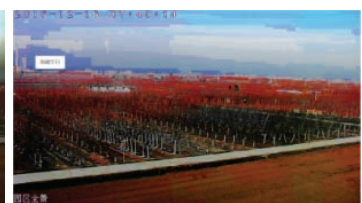

c3

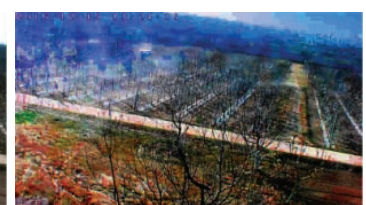

c4

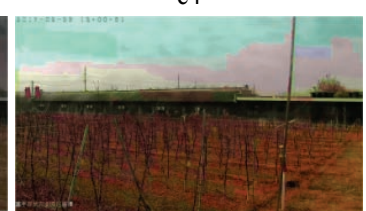

c5

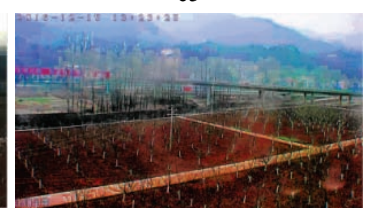

c6

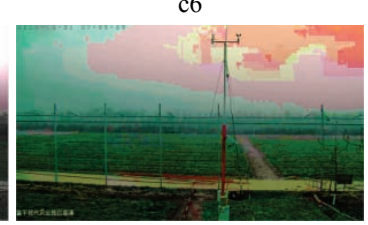

c7

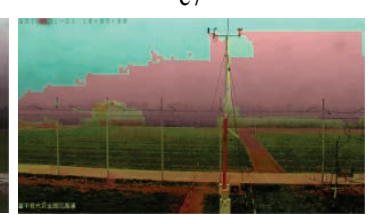

c8

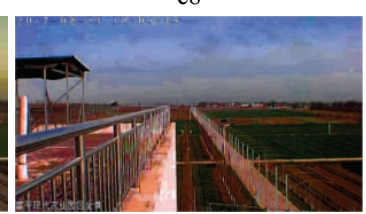

c9

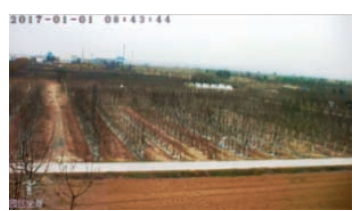

d1

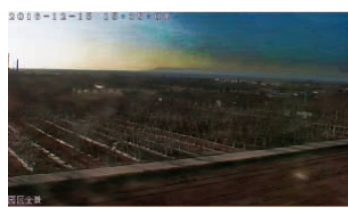

d2

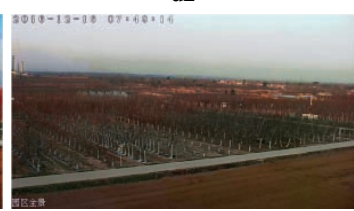

d3

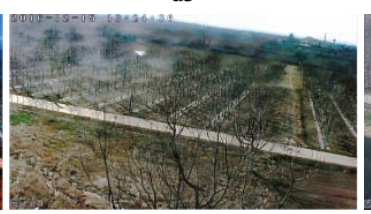

d4

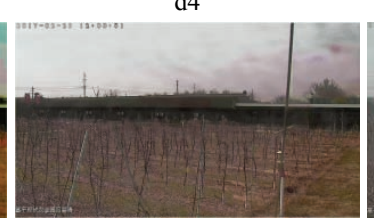

d5

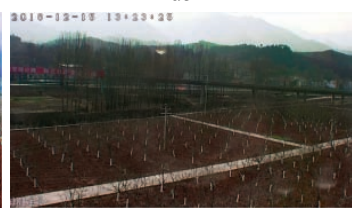

d6

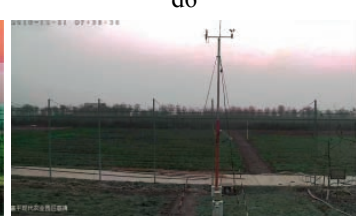

d7

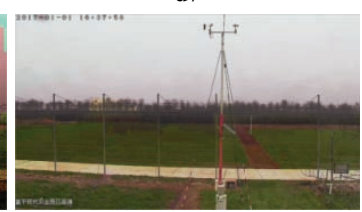

d8

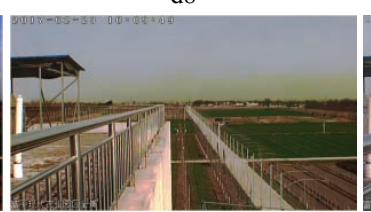

d9

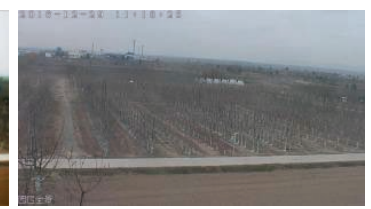

e1

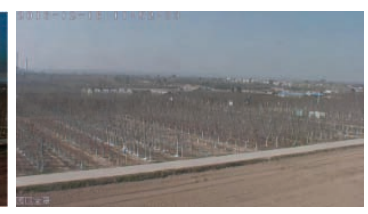

E2

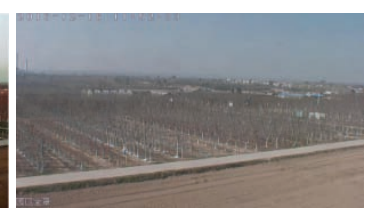

e3

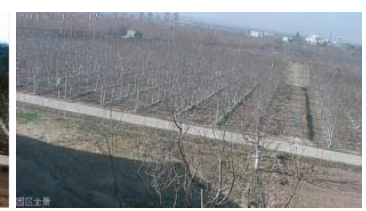

e4

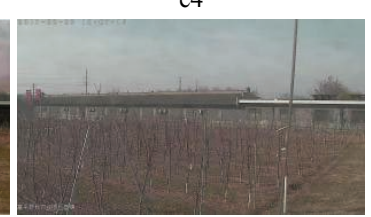

e5

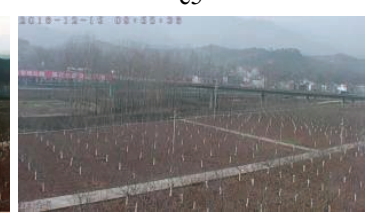

e6

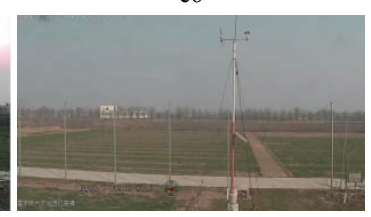

e7

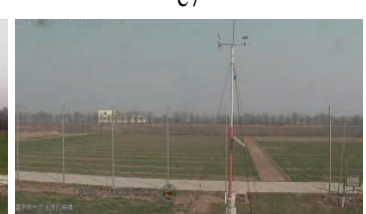

e8

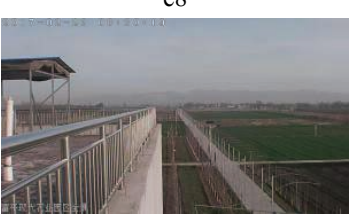

e9

Figure 4 Comparison of a1-a9 original images with fog, b1-b9 dehazed images based on DCP, c1-c9 dehazed images based on histogram equalization, d1-d9 dehazed images based on the proposed method, and e1-e9 reference images

As can be seen from Figure 5, the MSE obtained by histogram equalization was significantly larger than those for the other two methods, indicating that the image error using the histogram equalization method was large. The distortion on the images was obvious and histogram equalization cannot be directly used for image dehazing for agricultural field monitoring. 


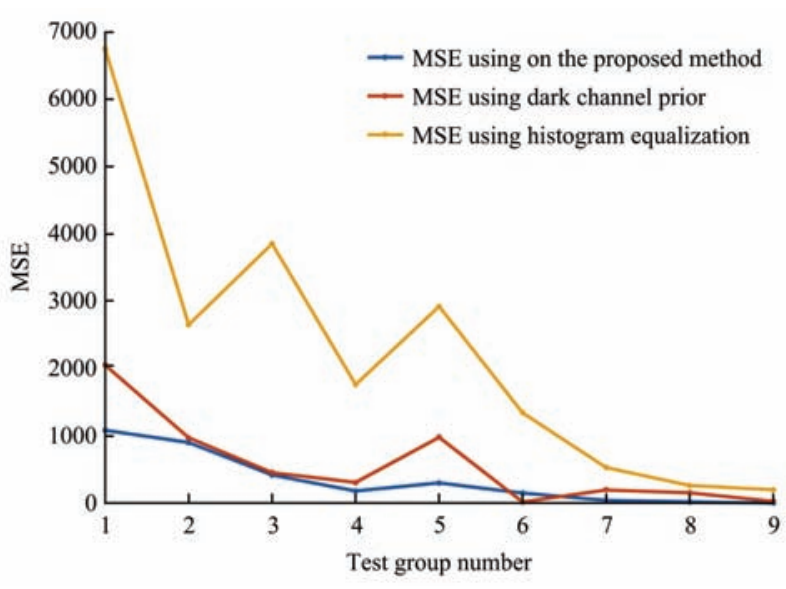

a. MSE

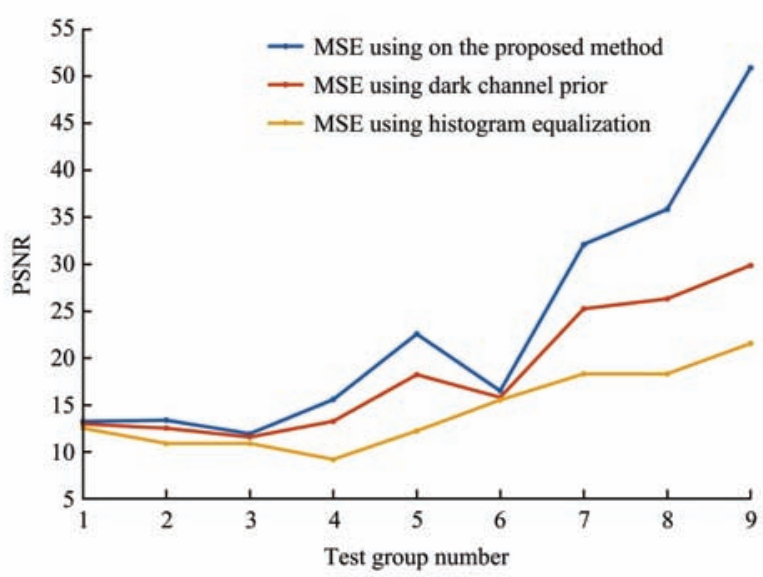

b. PSNR

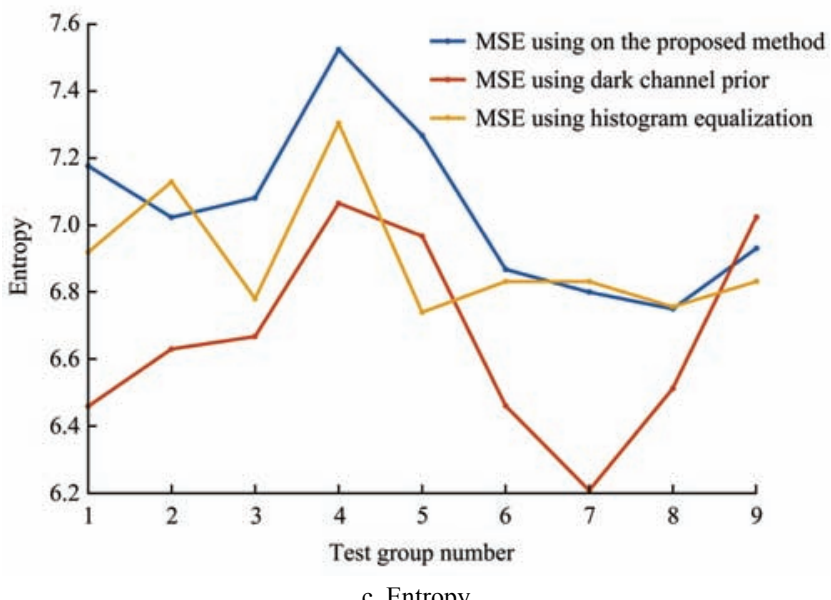

Figure 5 Evaluation of three dehazing methods using three indicators

\subsubsection{Effect of haze and light on image dehazing results}

As illustrated in the Figure 5, in general, the higher the haze level is, the higher the MSE and the lower the PSNR and Entropy of the dehazed images is, obtained by the three different methods. In addition, under the same condition, the MSE using on the proposed method is generally lower than the other two methods and PSNR and Entropy are generally higher.

For the first four groups of images, as air pollution in the Baishui station was more serious, the reference images and the images with fog were subjected to a high degree of noise interference. The dehazed images still had a large distortion so that the three indicators of the images did not reach the desired results.
For the sixth group of images, in addition to the influence from fog, the inference of the sunlight on the images was also great. After the image was brightened and the sky area was processed, the image looked more in line with the visual effect. However, the difference with the reference image increased, so the MSE for the proposed method was larger than that for the DCP method.

\section{Conclusions}

In order to solve the problems associated with traditional image dehazing methods for agricultural monitoring, an improved DCP algorithm was proposed in this study. With the improved method, the image distortion after processing was small. By comparing the processed images with the pre-selected reference images, it was found that the three evaluation indicators for the processed images obtained by this method were obviously improved. The main conclusions from this study are as follows:

1) The resulting image using the original DCP dehazing algorithm is usually dark with the distorted sky area. The proposed image dehazing method based on DCP and brightness enhancement can resolve the problems. The processed images have high contrast, uniform brightness, and natural-looking sky in line with human visual effect. The proposed method decreased MSE by an average of $40.07 \%$ and increased PSNR by an average of $21.81 \%$ and entropy by an average of $5.71 \%$ compared with the original DCP method. It outperformed the histogram equalization dehazing method with an average decrease of MSE by $84.78 \%$ and an average increase of PSNR by $38.95 \%$ and entropy by $2.04 \%$.

2) Serious haze will significantly increase the noise in the image and affect the image with fog. Therefore, the MSE between the resulting image and the reference image can vary. Sunlight will interfere with the fog, resulting in some differences between the reference image and the dehazed image.

\section{Acknowledgments}

This work was supported by the National High Technology Research and Development Program of China (863 Program) (No.2013AA10230402), Agricultural Science and Technology Project of Shaanxi Province (No. 2016NY-157) and Fundamental Research Funds Central Universities (2452016077). The authors wish to thank the developers of all the software and the authors of the papers cited in this research for their excellent work. Mention of trade names or commercial products in this article is solely for the purpose of providing specific information and does not imply recommendation or endorsement by the authors' employers.

\section{[References]}

[1] Wang Y P. Application of dynamic feature extraction in precision agriculture. China University of Science and Technology, 2015.

[2] Wu D, Zhu Q S. The latest research progress of image fogging. Journal of Automation, 2015; 2: 221-239.

[3] Han H F. Design of remote monitoring and management system for agricultural environmental information. Chinese Academy of Agricultural Sciences, 2009.

[4] Ren F D. Research on image de-hazing algorithm. Jilin University, 2015.

[5] Yu J, Xu D B, Liao Q M. Research progress of image de-hazing technology. Journal of Image and Graphics, 2011; 16(9): 1561-1576.

[6] Guo P, Cai Z X, Xie B, Tang J. Review and prospect of image de-hazing technology. Computer Application, 2010; 30(9): 2417-2421.

[7] Zhang Y L. An image-enhanced de-hazing method and its implementation. Electronic World, 2015(21): p. 106-107.

[8] Yang L C, Li B, Fan C, Jia C Q, Realization of Fast Foggy Algorithm 
Based on Image Enhancement. Electronic technology, 2015; 7: 30-32, 29.

[9] Li Y, Zhang Y F, Zhang Q, Geng A H, Chen J, Infrared Image Contrast Enhancement Based on De-hazing Model. Chinese laser, 2015(01): p. 306-314.

[10] Narasimhan S G, Nayar S K. Vision and the atmosphere. International Journal of Computer Vision, 2002; 48(3): 233-254.

[11] Nayar S K, Narasimhan S G. Vision in bad weather. in Proceedings of the International Conference on Computer Vision, IEEE Computer Society, 1999; Vol 2, p.820.

[12] He K, Sun J, Tang X. Single image haze removal using dark channel prior. IEEE Transactions on Pattern Analysis \& Machine Intelligence, 2010; 33(12): 2341-2353.

[13] Xiao C, Gan J. Fast image de-hazing using guided joint bilateral filter. The Visual Computer, 2012; 28(6-8): 713-721.

[14] Chen P F, Guo J K, Sung C C, Chang HH. An Improved Dark Channel-Based Algorithm for Underwater Image Restoration. 2014; 152: 311-316.

[15] Zhao Y, Yi C, Kong S G, Pan Q, Cheng Y. 3D Reconstruction and Dehazing with Polarization Vision[M]// Multi-band Polarization Imaging and Applications. Springer Berlin Heidelberg, 2016.

[16] Ansia S, Aswathy A L. Single Image Haze Removal Using White Balancing and Saliency Map. Procedia Computer Science, 2015; 46: 12-19.
[17] Graves N, Newsam S. Camera-based visibility estimation: Incorporating multiple regions and unlabeled observations. Ecological Informatics, 2014; 23: 62-68.

[18] Kumari A, Sahoo S K. Fast single image and video deweathering using look-up-table approach. AEU - International Journal of Electronics and Communications, 2015; 69(12): 1773-1782.

[19] Ni W, Gao X, Wang Y. Single satellite image de-hazing via linear intensity transformation and local property analysis. Neurocomputing, 2016; 175: 25-39.

[20] Hu Z, Liu Q, Zhou S, Huang M, Teng F. Image dehazing algorithm based on atmosphere scatters approximation model[C]// International Conference on Neural Information Processing. Springer-Verlag, 2012:159-168.

[21] Ding M, Tong R. Efficient dark channel based image de-hazing using quadtrees. Science China Information Sciences, 2013; 56(9): 1-9.

[22] Wang Y, Wu B. Improved single image de-hazing using dark channel prior. 2010 IEEE International Conference on Intelligent Computing and Intelligent Systems (ICIS), 2010.

[23] Wang J B, He N, Zhang L L, K Lu. Single image dehazing with a physical model and dark channel prior. Neurocomputing, 2015; 149(PB): 718-728.

[24] Hui H. Thin cloud-fog cover removed from remote sensing imagery based on stationary wavelet transformation. Atlantis Press, 2014. 